\title{
An Accurate Closed-Form Formula to Calculate the Dejittering Delay in Packetised Voice Transport
}

\author{
D. De Vleeschauwer ${ }^{1}$, G.H. Petit ${ }^{1}$, B. Steyaert ${ }^{2}$, S. Wittevrongel ${ }^{2}$ and H. Bruneel ${ }^{2}$ \\ ${ }^{1}$ Alcatel Bell, Corporate Research Center, \\ Francis Wellesplein 1, B-2018 Antwerpen, Belgium \\ \{danny.de_vleeschauwer, guido.h.petit\}@alcatel.be \\ ${ }^{2}$ Ghent University, Vakgroep TELIN, SMACS Research Group, \\ Sint-Pietersnieuwstraat 41, B-9000 Gent, Belgium \\ $\{b s$, sw, hb\} atelin.rug.ac.be
}

\begin{abstract}
The transport of voice over a packet-based network introduces jitter in the voice flow. The jitter is compensated in the dejittering buffer by artificially delaying the first packet of the flow over the so-called dejittering delay and then cyclically reading the packets from the dejittering buffer. In this paper, we first give a general numerical method to compute the dejittering delay. Then, we introduce and justify a closed-form heuristic formula to calculate the dejittering delay for a network consisting of identically loaded, independent nodes modelled as M/G/1 queues. We demonstrate the accuracy of the heuristic formula by means of some numerical examples. Since the heuristic formula is closed-form, accurate, and explicitly shows the influence of certain network parameters, it is the preferred method to dimension the dejittering buffer and to perform sensitivity studies.
\end{abstract}

\section{Introduction}

Although the feasibility of packet-based transport of real-time voice has been proven a long time ago, a lot of interest has risen lately in specific implementations: e.g., Voice and Telephony over ATM (VTOA) [1], Voice over Frame Relay (VoFR) [2], and especially Voice over IP (VoIP) [3,4,5,6,7,8,9].

In the packetised voice transport the voice signal is partitioned in equally sized intervals. Each interval is encoded and the corresponding code word is transported in a packet over the packet-based network. Hence, the packets are produced with a constant interdeparture time equal to the length of an interval. Constant bit-rate codecs produce code words of constant size. Codecs with voice activity detection produce code words of constant size during talk spurts and produce (practically) no packets during periods of silence.

In the packet-based network the packets of several flows compete for the available resources. Some packets can be processed immediately when they arrive at a network node, while others have to wait. Some packets need to wait longer than others, causing so-called jitter in the voice flow. Because the decoder needs the packets at the original constant rate, the jitter is compensated in the dejittering buffer by artificially delaying the first packet of a flow over the so-called dejittering delay and then 
cyclically reading the packets from the dejittering buffer $[8,9]$. The dejittering delay has to be carefully chosen. A too large a choice jeopardises the real-time delivery of the voice. A too small a choice results in a lot of packets arriving in the dejittering buffer after they were supposed to be read, which means that they are effectively lost.

In this paper we develop a heuristic formula to calculate the dejittering delay to compensate for the jitter introduced in a network dedicated to the transport of voice, i.e., free of data. For voice transported over a packet-based network simultaneously carrying data, the heuristic formula is of some use too (see $[8,9])$.

In the following sections, the network nodes traversed by a given voice flow will be modelled as a sequence of independent M/G/1 queues. This choice can be motivated as follows. First, note that for a given voice flow, packets enter the network at a constant rate (possibly with some gaps in between in case of voice activity detection), which implies that modelling these voice packet arrival streams at the network's ingress by a Poisson process is actually a worst-case scenario. In [10], it has now been argued that if a number of flows that are 'better than Poisson' (i.e., with lower variability of the interarrival times) are multiplexed in a FIFO buffer, then the outgoing streams continue to be better than Poisson. Thus it is clear that this property will be propagated throughout the subsequent network nodes visited by a flow, provided that these nodes can be regarded as being independent. This will be the case if a given flow accounts only for a small fraction of the total amount of traffic in a node (see e.g. [11]) which is the case in a (dedicated) packet-based VoIP network, where a large number of low bit rate voice flows are to be multiplexed in each node.

In the next section, the mathematical model to determine the dejittering delay for a dedicated packet-based voice network is described. Section 3 introduces and justifies a closed-form heuristic formula to calculate the dejittering delay. In section 4 , the performance of this heuristic formula is demonstrated with some numerical results. In the last section some conclusions are drawn.

\section{The Dejittering Delay for a Network of M/G/1 Queues}

In order to transport interactive voice over a packet-based network, the voice signal is first encoded and the corresponding code words are transported in packets of constant size. Such a flow of packets carrying voice requires real-time delivery; i.e., the packets have to arrive at the destination within a certain delay bound. In each node of the network the packets of the flow experience a delay, consisting of a deterministic service time (determined by the packet size) and a stochastic waiting time. The stochastic waiting time introduces jitter in the voice flow. Since the voice decoder requires the packets at a constant rate, this jitter has to be compensated.

In a network of $\mathrm{M} / \mathrm{G} / 1$ queues the largest possible total waiting time is infinite. When the $(1-P)$-quantile $w_{P}$ is used as dejittering delay a fraction $P$ of the packets are lost in the dejittering buffer. Fortunately, voice codecs can tolerate some packet loss. Depending on the codec a packet loss in the range $\left[10^{-5}, 10^{-2}\right]$ can be tolerated.

Consider a route through a dedicated VoIP network (i.e., a network free of data) traversing $N$ identically loaded, independent nodes modelled as M/G/1 queues. The service discipline in each node is FIFO, because all packets in the network require real-time delivery, and hence, have the same priority. The probability density function 
(pdf) $p_{w}(w)$ of the waiting time $W$ introduced in the network is known under the form of the Laplace transform

$$
W(s)=E\left\{e^{-s W}\right\}=\int d x p_{W}(x) e^{-s x} .
$$

We use the following definitions. The load of the voice traffic is $\rho$. The Laplace transform of the pdf of the service time required by a voice packet is $B(s)$. The $k$-th moment of this service time is denoted as $b_{k}$. The Laplace transform of the pdf of the residual service time, which is the remaining service time of the voice packet in service observed by an arriving voice packet, is

$$
R(s)=\frac{1-B(s)}{b_{1} s} .
$$

With these definitions, the Laplace transform of the waiting time pdf introduced in a single node, modelled as an M/G/1 queue, is given by [12]

$$
W_{1}(s)=\frac{1-\rho}{1-\rho R(s)} .
$$

In the introduction, it was argued that for the case of a network consisting of multiple identical nodes, the waiting times encountered in consecutive nodes can be regarded as being statistically independent. Hence, if we denote by $W_{N}$ the total waiting time encountered through $N$ nodes, its pdf is obtained from the $N$-fold convolution of the individual waiting time's pdf, which yields for the corresponding Laplace transform

$$
W_{N}(s)=\left[W_{1}(s)\right]^{N}
$$

The tail distribution of the total waiting time $W_{N}$ is defined as

$$
\operatorname{Pr}\left[W_{N}>w\right]=t_{W}(w)=\int_{w}^{\infty} d x p_{W}(x),
$$

where $p_{W}(x)$ is the inverse of the Laplace transform (4). This transform can be inverted numerically by using a Fast Fourier Transform (FFT). The major disadvantages of this approach are that it requires a lot of computation time and that it becomes errorprone for low values of $P$. If the calculations are performed in single precision, the influence of the rounding errors introduced in this procedure is already noticeable for $P$-values around $10^{-4}$. For smaller $P$-values rounding errors have too large an influence to lead to meaningful results. Therefore, we propose the analytic/heuristic approach described in the following sections. Our objective is to derive a closed-form formula for the (1-P)-quantile $w_{P}$ of the total waiting time from transform (4). 


\section{A Heuristic Formula}

In this section we introduce (see [13]) and justify a heuristic formula, to determine the $(1-P)$-quantile $w_{P}$ of the total waiting time. The heuristic formula states that the $(1-P)$ quantile is equal to the sum of the average total waiting time $\mu_{N}$ and a number of times the standard deviation $\sigma_{N}$ of the total waiting time:

$$
w_{P}=\mu_{N}+\alpha_{N}(P) \sigma_{N} .
$$

The value of $\alpha_{N}(P)$ depends solely on the number $N$ of nodes traversed and the value of $P$, but is independent of the service time characteristics of a voice packet. The factor $\alpha_{N}(P)$ is chosen such that if the waiting time in 1 node were exponentially distributed, the heuristic formula (6) would be exact. It follows that

$$
\alpha_{N}(P)=\frac{E_{N}^{-1}(P)-N}{\sqrt{N}},
$$

where

$$
E_{N}(w)=\int_{w}^{\infty} d x\left[\frac{x^{N-1}}{(N-1) !} \exp (-x)\right]=\exp (-w) \sum_{j=0}^{N-1} \frac{w^{j}}{j !},
$$

is the tail distribution of the normalised Erlang distribution of degree $N$, which is equal to the $\chi^{2}$-distribution with $2 N$ degrees of freedom. This latter function and its inverse are included in standard spreadsheets, e.g. Excel. Hence, formula (6) is closed-form. The values $\alpha_{N}(P)$ are tabulated in Table 1 . In the next subsections, we justify the heuristic formula (6) and determine its range of applicability.

Table 1. The weighting factor $\alpha_{N}(P)$.

\begin{tabular}{|c||c|c|c|c|c|c||c|c|c||c|}
\hline$\alpha_{N}(P)$ & $N=1$ & $N=2$ & $N=4$ & $N=8$ & $N=16$ & $N=32$ & $N=2^{7}$ & $N=2^{10}$ & $N=2^{13}$ & $N \rightarrow \infty$ \\
\hline \hline$P=10^{-2}$ & 3.605 & 3.280 & 3.023 & 2.828 & 2.686 & 2.582 & 2.455 & 2.372 & 2.342 & 2.327 \\
\hline$P=10^{-3}$ & 5.908 & 5.115 & 4.531 & 4.111 & 3.811 & 3.599 & 3.344 & 3.180 & 3.122 & 3.091 \\
\hline$P=10^{-4}$ & 8.210 & 6.899 & 5.957 & 5.290 & 4.821 & 4.493 & 4.102 & 3.853 & 3.766 & 3.719 \\
\hline$P=10^{-5}$ & 10.513 & 8.653 & 7.333 & 6.407 & 5.762 & 5.312 & 4.781 & 4.445 & 4.328 & 4.265 \\
\hline$P=10^{-6}$ & 12.816 & 10.386 & 8.675 & 7.482 & 6.654 & 6.080 & 5.404 & 4.980 & 4.833 & 4.753 \\
\hline$P=10^{-7}$ & 15.118 & 12.106 & 9.993 & 8.526 & 7.511 & 6.809 & 5.987 & 5.473 & 5.296 & 5.199 \\
\hline$P=10^{-8}$ & 17.421 & 13.814 & 11.292 & 9.545 & 8.340 & 7.509 & 6.538 & 5.933 & 5.725 & 5.612 \\
\hline$P=10^{-9}$ & 19.723 & 15.514 & 12.577 & 10.546 & 9.147 & 8.185 & 7.063 & 6.367 & 6.127 & 5.997 \\
\hline$P=10^{-10}$ & 22.026 & 17.207 & 13.850 & 11.530 & 9.936 & 8.840 & 7.566 & 6.778 & 6.507 & 6.361 \\
\hline
\end{tabular}

\subsection{Asymptotic-Tail Approximation}

For practical Laplace transforms $B(s)$ of the service time pdf, $W_{1}(s)$ has a clear dominant pole $p$, i.e., a real pole with multiplicity 1 and an absolute value (much) 
smaller than the absolute values of the other poles. This implies that eq. (3) can be written as

$$
W_{1}(s)=\frac{F(s)}{(1+\lambda s)}
$$

with $\lambda=-1 / p$ and $F(s)$ an analytical function in the neighbourhood of the dominant pole $p$. Due to eq. (3), the pole $p$ satisfies $1-\rho R(p)=0$. We refer to $\lambda$ as the rate of decay of the tail.

By using the residue theorem [12], we can approximate the pdf of the waiting time in a network consisting of 1 node as

$$
p_{W}(x) \approx \lim _{s \rightarrow p}\left[\frac{1}{\lambda} F(s) \exp (s x)\right]=\frac{1}{\lambda} \exp \left(-\frac{x+\delta}{\lambda}\right)
$$

with

$$
\delta=\frac{\ln (F(p))}{p}
$$

Eq. (10) is asymptotically exact, i.e., for sufficiently large values of $x$. Hence, the (1$P$ )-quantile of the waiting time in 1 node is given by

$$
w_{P}=\lambda E_{1}^{-1}(P)-\delta
$$

For a network consisting of $N$ nodes, the multiplicity of the dominant pole $p$ is $N$. In this case, use of the residue theorem [12] leads to the following approximation for the pdf of the total waiting time:

$$
p_{W}(x) \approx \lim _{s \rightarrow p}\left\{\frac{1}{(N-1) !} \frac{d^{N-1}}{d s^{N-1}}\left[\left(\frac{1}{\lambda}\right)^{N}(F(s))^{N} \exp (s x)\right]\right\} .
$$

Because of the derivative appearing in eq. (13), the case of $N$ nodes is considerably more difficult to handle than the case of 1 node. Inspired by eq. (11) we now state that

$$
F(s)=\exp (\delta s)
$$

in the neighbourhood of the dominant pole $p$. As seen above, this assumption leads to the correct result for the case $N=1$. Intuitively, it is expected that for low values of $N$, this approximation will be reasonably good, but when $N$ increases the errors introduced by this approximation will become larger. With the approximation (14) the calculation of the residue of eq. (13) is trivial, and hence, the tail distribution can easily be calculated as

$$
t_{W}(w)=E_{N}\left(\frac{w+N \delta}{\lambda}\right)
$$


The (1-P)-quantile is then given by

$$
w_{P}=\lambda E_{N}^{-1}(P)-N \delta,
$$

which is the multi-node extension of eq. (12). We refer to eq. (16) as 'the formula based on the method of the dominant pole', meaning that we use the exact (numerically calculated) values of $\lambda$ and $\delta$. It is only a good approximation if there is a clear dominant pole and if the number of nodes in the network $N$ is low. Since eq. (16) requires the exact knowledge of the dominant pole $p$, which involves a root finding method to calculate $p$ numerically, this formula is not closed-form. Therefore, we attempt to express $p$ in terms of known quantities.

\subsection{Heavy-Load Approximation}

In order to obtain a closed-form formula, we introduce a heavy-load approximation for $\lambda$ and $\delta$. This is done as follows. Under heavy load $\rho$, the Laplace transform of eq. (3) can be approximated by

$$
W_{1}(s) \approx \frac{1}{1+A s-\tau \frac{(A s)^{2}}{2}},
$$

with

$$
A=\frac{\rho}{1-\rho} \frac{b_{2}}{2 b_{1}} \quad ; \quad \tau=\frac{\rho}{1-\rho} \frac{b_{3}}{3 b_{1}} \frac{1}{A^{2}} .
$$

Notice that as $\rho$ tends to $1, \tau$ tends to 0 . Therefore, in the following, we neglect terms of the order of $\tau^{2}$. Also note that the parameters $A$ and $\tau$ are closely related to the average $\mu_{1}$ and the standard deviation $\sigma_{1}$ of the waiting time in a single node:

$$
\mu_{1}=A \quad ; \quad \sigma_{1}=A \sqrt{1+\tau} \approx A\left(1+\frac{\tau}{2}\right) .
$$

With the approximation (17) for $W_{1}(s)$ the rate of decay of the tail is given by

$$
\lambda=\frac{A \tau}{\sqrt{1+2 \tau}-1} \approx A\left(1+\frac{\tau}{2}\right) \approx \sigma_{1} .
$$

This states that the rate of decay of the tail $\lambda$ can be very well approximated by the standard deviation $\sigma_{1}$ of the waiting time in 1 node.

Furthermore, (still under approximation (17)) we readily see from

$$
\lim _{s \rightarrow p}(1+\lambda s) W_{1}(s)=\frac{\tau}{1+2 \tau-\sqrt{1+2 \tau}},
$$


that

$$
\delta=\lambda \ln \left(\frac{1+2 \tau-\sqrt{1+2 \tau}}{\tau}\right) \approx \frac{\lambda \tau}{2} \approx \sigma_{1}-\mu_{1}
$$

With the approximations (20) and (22) for $\lambda$ and $\delta$, eq. (16) can easily be rewritten in the form of the heuristic formula of eq. (6).

\subsection{Large-Network Approximation}

If the number of nodes traversed is large, the approximation of eq. (14) no longer holds and the formula based on the method of the dominant pole (eq. (16)) becomes inaccurate. In that case, however, the law of large numbers justifies the heuristic formula (6). When a large number $N$ of nodes is traversed the pdf of the total waiting time tends to a Gaussian with average $\mu_{N}$ and standard deviation $\sigma_{N}$. In this case, the tail distribution is approximated by

$$
t_{W}(w)=\operatorname{erfc}\left(\frac{w-\mu_{N}}{\sigma_{N}}\right)=\frac{1}{\sqrt{2 \pi}} \int_{\frac{w-\mu_{N}}{\sigma_{N}}}^{\infty} d x \exp \left(-\frac{x^{2}}{2}\right) .
$$

Hence, the (1-P)-quantile is given by

$$
w_{P}=\mu_{N}+\operatorname{erfc}^{-1}(P) \sigma_{N} .
$$

Since the normalised Erlang distribution of degree $N$ (see eq. (8)) converges to a Gaussian distribution with average value and variance both equal to $N$ as $N$ tends to infinity, we can immediately conclude that (see eq. (7))

$$
\lim _{N \rightarrow \infty} \alpha_{N}(P)=\operatorname{erfc}^{-1}(P)
$$

This behaviour can also be observed in Table 1. Hence, eq. (24) is clearly of the form of the heuristic formula (6).

\section{Numerical Results}

In this section the performance of the heuristic formula is demonstrated with some numerical examples. We consider the $M / D / 1$ queue and $M / D_{1}+D_{2} / 1$ queue.

The M/D/1 queue may be used for an IP network transporting voice where all voice codecs produce flows of packets of the same size. In this case, all voice packets have the same deterministic service time, and the corresponding Laplace transform is given by

$$
B(s)=\exp (-s)
$$


Remark that the average service time is normalised, i.e. $b_{1}=1$. When $N$ nodes are traversed, the average $\mu_{N}$ and standard deviation $\sigma_{N}$ of the total waiting time are:

$$
\begin{gathered}
\mu_{N}=N\left(\frac{\rho}{2(1-\rho)}\right) ; \\
\sigma_{N}=\sqrt{N} \sqrt{\left(\frac{\rho}{2(1-\rho)}\right)^{2}+\frac{\rho}{3(1-\rho)}} .
\end{gathered}
$$

The $M / D_{1}+D_{2} / 1$ queue may e.g. be used for an IP network transporting voice, where the voice flows are produced by one of two types of codecs: e.g. a codec producing $64 \mathrm{~kb} / \mathrm{s}$ and a low bit rate codec producing $8 \mathrm{~kb} / \mathrm{s}$. Because in delay sensitive services (e.g. voice services) the end-to-end delay is limited (to e.g. $150 \mathrm{~ms}$ ), the packetisation delay, i.e., the time to fill a packet, is limited too. Therefore, the packets for the $64 \mathrm{~kb} / \mathrm{s}$ codec are likely to be larger than the packets for the $8 \mathrm{~kb} / \mathrm{s}$ codec. Because on the one hand also queueing delay and dejittering delay and on the other hand also the header size play a role, the packet size ratio will not be exactly 8 to 1 , but slightly less. Here, we take a ratio of 7 to 1 as an example. Furthermore, we assume that half of the flows use the $8 \mathrm{~kb} / \mathrm{s}$ codec, the other half use the $64 \mathrm{~kb} / \mathrm{s}$ codec. Hence, the Laplace transform of the pdf of the service time is

$$
B(s)=0.5 \exp (-0.25 s)+0.5 \exp (-1.75 s) \quad .
$$

Remark that again the average service time is normalised, i.e. $b_{1}=1$. When $N$ nodes are traversed, the average $\mu_{N}$ and standard deviation $\sigma_{N}$ of the total waiting time are:

$$
\begin{gathered}
\mu_{N}=N\left(\frac{\rho}{2(1-\rho)} 1.5625\right) ; \\
\sigma_{N}=\sqrt{N} \sqrt{\left(\frac{\rho}{2(1-\rho)} 1.5625\right)^{2}+\frac{\rho}{3(1-\rho)} 2.6875} .
\end{gathered}
$$

The accuracy of the approximation for the rate of decay of the tail $\lambda$ introduced in eq. (20) is illustrated in Figure 1 and Figure 3. These figures show that for sufficiently large loads the rate of decay of the tail $\lambda$ is very well approximated by the standard deviation $\sigma_{1}$ of the waiting time in 1 node.

Figure 2 and Figure 4 show how the dejittering delay increases as the load $\rho$ increases for a flow traversing $N$ nodes modelled as $\mathrm{M} / \mathrm{D} / 1$ queues and $\mathrm{M} / \mathrm{D}_{1}+\mathrm{D}_{2} / 1$ queues respectively. The dejittering delay associated with several $P$-values is calculated with the method of the dominant pole (eq. (16)) and with the heuristic formula (eq. (6)). For $P=10^{-2}$ and $P=10^{-3}$ the exact dejittering delay (obtained via the FFT) is also given. For smaller values of $P$, the FFT-based method becomes numerically unstable, and the use of an efficient alternative method to calculate the dejittering delay, such as the one introduced in this paper, becomes mandatory. It can be seen that both methods produce accurate results for all values of $N$ and $P$, if the 


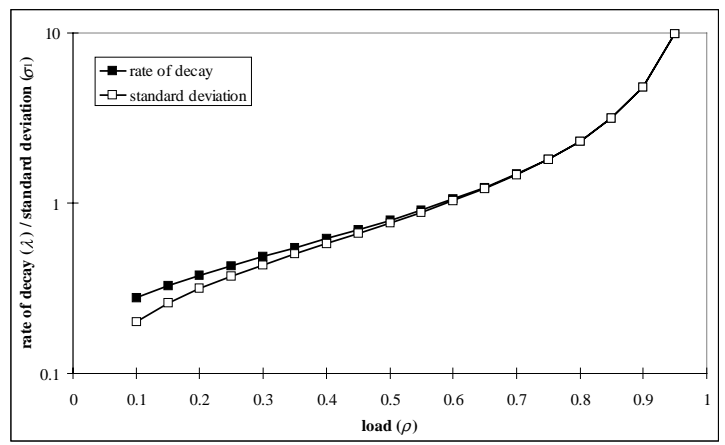

Fig. 2. The rate of decay of the tail $(\lambda)$ and the standard deviation of the waiting time $\left(\sigma_{1}\right)$ for the $\mathrm{M} / \mathrm{D} / 1$ queue.
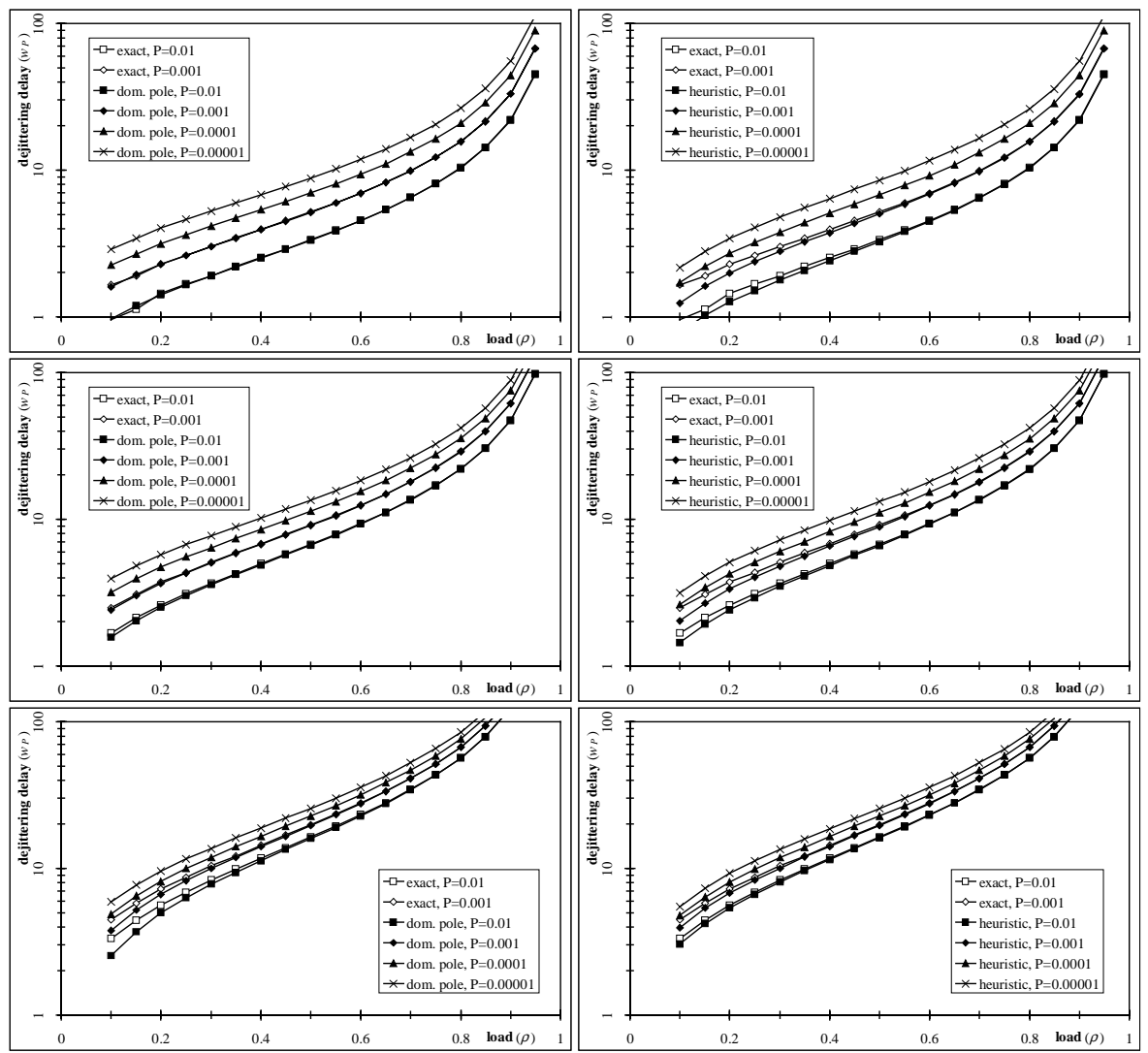

Fig. 2. The dejittering delay associated with several values of the packet loss $P$ for a network consisting of $N \mathrm{M} / \mathrm{D} / 1$ nodes. The number of nodes $N$ is 1 (top), 4 (middle), or 16 (bottom). The method of the dominant pole (left) is compared with the heuristic formula (right). The exact solution (obtained via the FFT) is also given for large $P$ values (0.01 and 0.001). 
load $\rho$ is above 0.5 . For networks consisting of a moderate amount of nodes $(N \leq 4)$, the method of the dominant pole slightly outperforms the heuristic formula. For large networks the heuristic formula is slightly better. This behaviour can be explained as follows. For a network consisting of 1 node the only approximation the method of the dominant pole makes is that it neglects the influence of the poles other than the dominant pole. The heuristic formula further introduces approximation (20), i.e., it does not use the exact rate of decay of the tail, but approximates it by the standard deviation of the waiting time in 1 node. Hence, in a network consisting of 1 node the heuristic formula is outperformed by the method of the dominant pole. For a large network, on the other hand, the method of the dominant pole also has to introduce approximation (14). Again the heuristic formula further introduces approximation (20). Apparently, both approximations (14) and (20) roughly compensate each other, if the number of network nodes becomes large. This compensation effect is justified by the large-network approximation of Section 3.3. Hence, for the case of a large network the heuristic formula becomes more accurate than the method of the dominant pole.

Remark by comparing Figure 4 with Figure 2 that the dejittering delay for a network of $M / \mathrm{D}_{1}+\mathrm{D}_{2} / 1$ nodes is about $60 \%$ higher than the dejittering delay for a network of $\mathrm{M} / \mathrm{D} / 1$ nodes. With the heuristic formula this can be readily concluded by comparing the formulae for the average delay and standard deviation of the delay (eq. (27) with eq. (30) and eq. (28) with eq. (31)). To be able to draw the same conclusion with the FFT-based method or the method of the dominant pole the curves of Figure 2 and Figure 4 have to be explicitly computed.

\section{Discussion and Conclusions}

With the theoretical justification of Section 3 and the numerical examples of Section 4, we conclude that the heuristic formula (6)

1. explicitly shows the influence of certain parameters: the dejittering delay is the sum of the average total waiting time and a number of times the standard deviation of the total waiting time; the weight of the standard deviation only depends on the number of nodes traversed and the fraction of packets that (are allowed to) arrive too late in the dejittering buffer;

2. slightly underestimates the dejittering delay at low loads;

3. produces very accurate results for all values of $N$ and $P$ when the load is larger than 0.5 ; and

4. is slightly outperformed by the method of the dominant pole (16) for a network consisting of a moderate amount of nodes, but outperforms the method of the dominant pole for large networks.

Furthermore, having a closed-form formula for the dejittering delay has the advantage that it explicitly shows how the dejittering delay increases as the variability of the service time increases $\left(b_{2}\right.$ and $b_{3}$ increase while keeping $\left.b_{1}=1\right)$. Also we readily observe that the dejittering delay for a network consisting of $N$ nodes is much smaller than $N$ times the dejittering delay for 1 node. Estimating the dejittering delay for a network consisting of $N$ nodes by taking $N$ times the dejittering delay for 1 node leads to a large overestimation of the dejittering delay. 


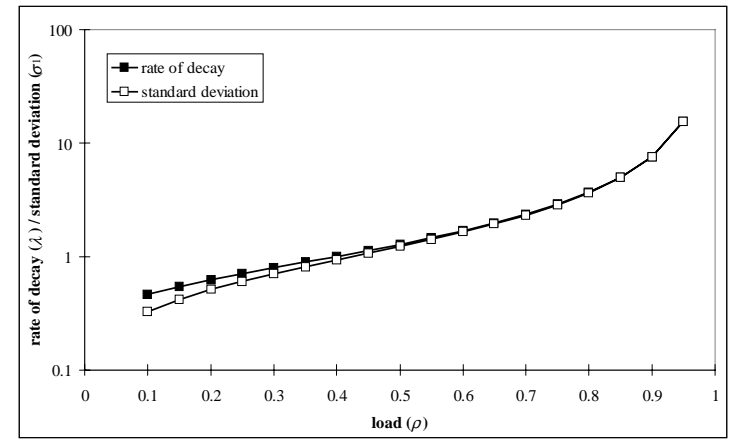

Fig. 4. The rate of decay of the tail $(\lambda)$ and the standard deviation of the waiting time $\left(\sigma_{1}\right)$ for the $\mathrm{M} / \mathrm{D}_{1}+\mathrm{D}_{2} / 1$ queue.
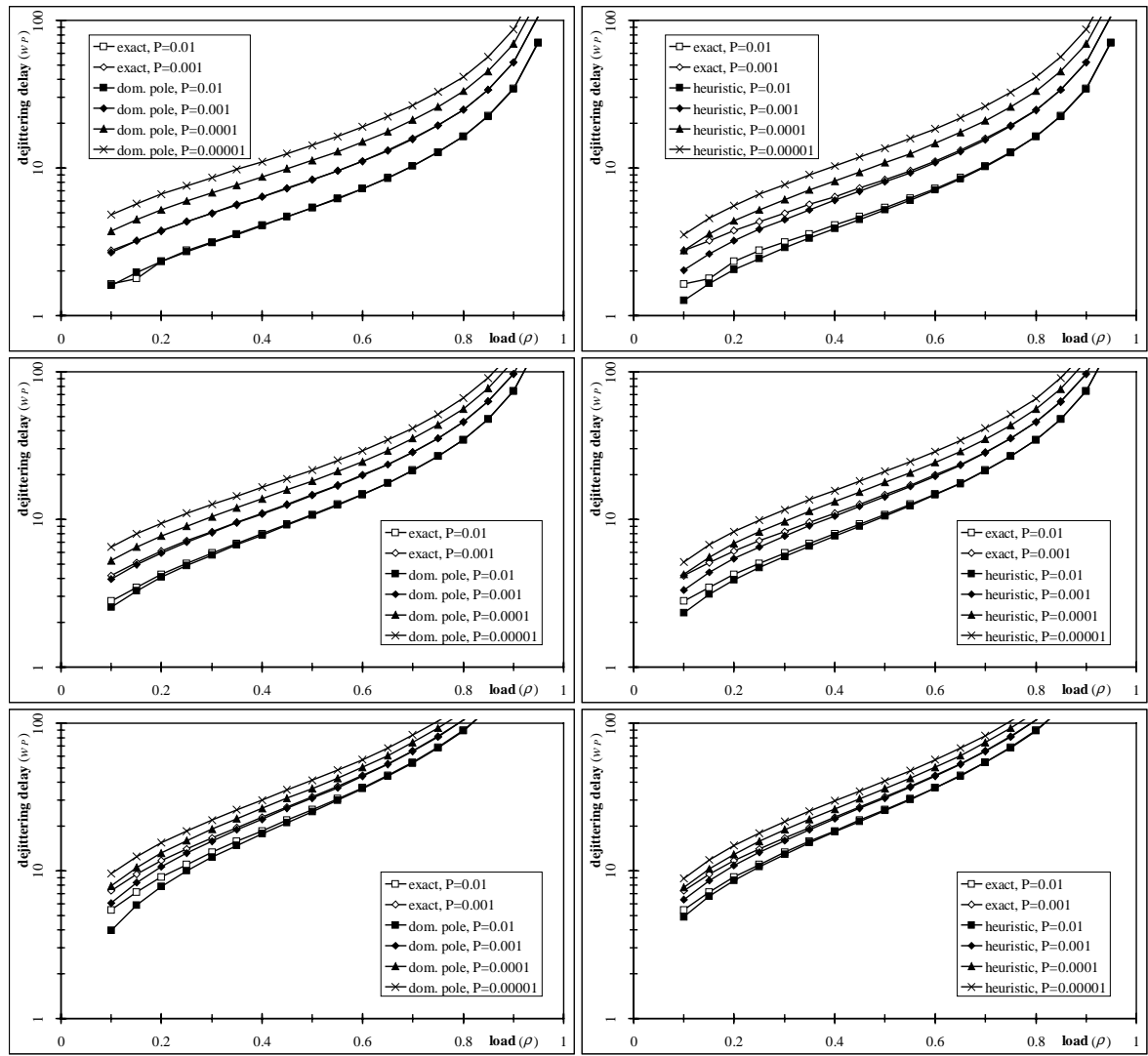

Fig. 4. The dejittering delay associated with several values of the packet loss $P$ for a network consisting of $N \quad \mathrm{M} / \mathrm{D}_{1}+\mathrm{D}_{2} / 1$ nodes. The number of nodes $N$ is 1 (top), 4 (middle) or 16 (bottom). The method of the dominant pole (left) is compared with the heuristic formula (right). The exact solution (obtained via the FFT) is also given for large $P$-values $(0.01$ and 0.001$)$. 


\section{Acknowledgements}

This work was carried out within the framework of the project LIMSON sponsored by the Flemish Institute for the Promotion of Scientific and Technological Research in the Industry (IWT). The fourth author is a postdoctoral fellow of the Fund for Scientific Research - Flanders (Belgium) (F.W.O.).

\section{References}

1. D.J. Wright, "Voice over ATM: An Evaluation of Implementation Alternatives", IEEE Communications Magazine, Vol. 34, No. 5, pp. 72-80, May 1996.

2. C. Bucholtz, "Another Avenue for Voice", Telephony, pp. 60, May 1997.

3. A. Cray, "Voice over IP, Hear's how", Data Communications, pp. 44-58, Apr. 1998.

4. R. Gareiss, "Voice over IP Services: The Sound Decision", Data Communications, pp. 7584, Mar. 1998.

5. T.J. Kostas, M.S. Borella, I. Sidhu, G.M. Schuster, J. Grabiec, J. Mahler, "Real-Time Voice over Packet-Switched Networks", IEEE Network, pp. 18-27, Jan./Feb. 1998.

6. T. Lewis, "VoIP; Killer App for the Internet", IEEE Internet Computing, pp. 110-112, Nov.Dec. 1997.

7. S.R. Ahuja, K.G. Murti, "Packet Telephony”, Bell Labs Technical Journal, pp. 5-14, Spring 1997.

8 K. Van Der Wal, M. Mandjes, H. Bastiaansen, "Delay Performance Analysis of the New Internet Services with Guaranteed QoS", Proceedings of the IEEE, Vol. 85, No. 12, pp. 1947-1957, Dec. 1997.

9. M. Mandjes, K. Van Der Wal, R. Kooij, H. Bastiaansen, "End-to-end Delay Models for Interactive Services on Large Scale IP Networks", Proceedings of IFIP'99 (Antwerp, Belgium, 28-30 June 1999).

10. F. Brichet, L. Massoulié, J.W. Roberts, "Stochastic Ordering and the Notion of Negligible CDV”, Proceedings of ITC'15 (Washington, June 1997), pp. 1433-1444.

11. W.-C. Lau, S.-Q. Li, "Traffic Distortion and Inter-source Cross-correlation in High-speed Integrated Networks”, Computer Networks and ISDN Systems, Vol. 29, pp. 811-830, 1997.

12. L. Kleinrock, "Queueing Systems, Vol. 1: Theory", John Wiley and Sons, New York, 1975.

13. G.H. Petit, “A BASIC Tool to Calculate Jitter”, BASIC program, Personal communication, 1988. 